\title{
Pituicytoma of the Neurohypophysis: Analysis of Cell Proliferation Biomarkers
}

\author{
J. Karamchandani, L. V. Syro, H. Uribe, E. Horvath, K. Kovacs
}

Can J Neurol Sci. 2012; 39: 835-837

A 37-year-old man presented with a one year history of diminished libido and a six month history of poor concentration and headaches. No visual field changes were identified. Laboratory testing revealed normal blood hormone levels of total testosterone $(10.16 \mathrm{nmol} / \mathrm{L}$, normal 6.7-28.9), growth hormone $(0.0 \mathrm{ng} / \mathrm{ml}$, normal<5.0), insulin-like growth factor (132 ng/ml, normal 48-255), thyroid stimulating hormone (2.48 $\mathrm{mUI} / \mathrm{ml}$, normal $0.5-6.0)$, free thyroxin $(0.98 \mathrm{ng} / \mathrm{dl}$, normal 0.8 $1.8)$, and cortisol $(8.7 \mathrm{mcg} / \mathrm{ml}$, normal 5-25). Blood prolactin levels were mildly elevated $(131 \mathrm{ng} / \mathrm{ml}$, normal $0-15)$, with decreased blood levels of luteinizing hormone $(0.09 \mathrm{mUI} / \mathrm{ml}$, normal 2-12), and follicle stimulating hormone $(0.0 \mathrm{mUI} / \mathrm{ml}$, normal 1-8).

Magnetic resonance imaging (MRI) revealed a circumscribed suprasellar lesion measuring 25×25mm (Figure 1) displacing the pituitary stalk and compressing the optic chiasm and the floor of the third ventricle. There was no evidence of hydrocephalus. Based on MRI a diagnosis of pituicytoma was made. In order to get tissue for morphological studies and to resect subtotally the lesion a right fronto-temporal craniotomy was performed. At surgery a soft, highly vascular lesion was identified in the region of the neurohypophysis which was partially resected. Gross total resection was not attempted in order to avoid iatrogenic neuroendocrine deficits. The post-operative evolution was uneventful.

The resected specimen was fixed in formalin and embedded in paraffin. Hematoxylin and eosin (H\&E) stained sections demonstrated a proliferation of monotonous spindled cells with round to ovoid nuclei and modest eosinophilic cytoplasm (Figure 2). Mitotic figures were not identified. The tumor contained scattered admixed lymphocytes and macrophages. No Herring bodies, Rosenthal fibers, eosinophilic granular bodies, or oncocytic appearing cells were apparent. The tumor cells were PAS negative.

For immunohistochemistry four micrometer sections from the formalin-fixed paraffin embedded tissue were stained with the streptavidin-biotin-peroxidase method. The tumor cells were strongly positive for S-100 protein (highlighting both tumor cell nuclei and cytoplasm). Glial fibrillary acidic protein (GFAP) showed patchy positivity throughout the tumor. Vimentin and vascular endothelial growth factor (VEGF) were diffusely

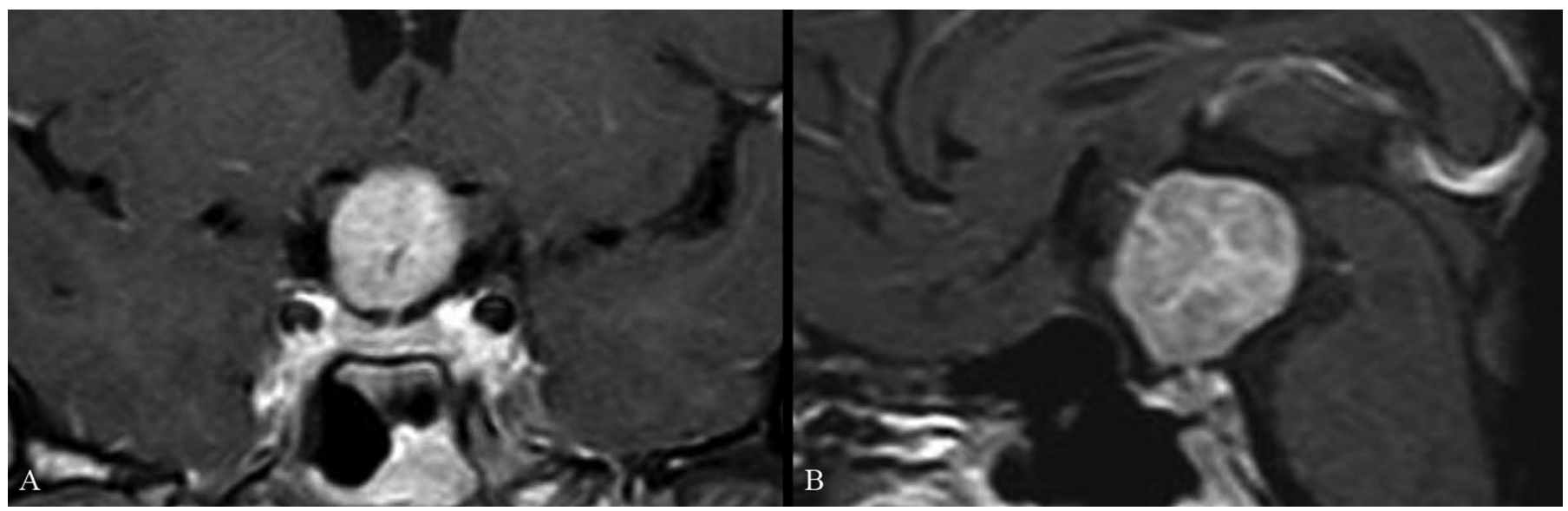

Figure 1: Coronal (A) and Sagittal (B) T1-weighted MRI with gadolinium enhancement showing a round suprasellar lesion with compression of the optic chiasm and the floor of the third ventricle.

From the Department of Pathology and Laboratory Medicine (JK, EH, KK), St. Michaels Hospital; Department of Laboratory Medicine and Pathobiology (JK, KK), University of

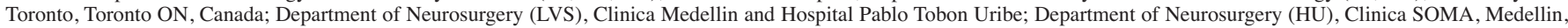
Colombia.

Received March 15, 2012. Final Revisions Submitted June 19, 2012.

Correspondence to: Jason Karamchandani, St. Michael's Hospital, 30 Bond Street, Toronto, Ontario, M5B 1W8, Canada. Email: jkaramch@gmail.com. 


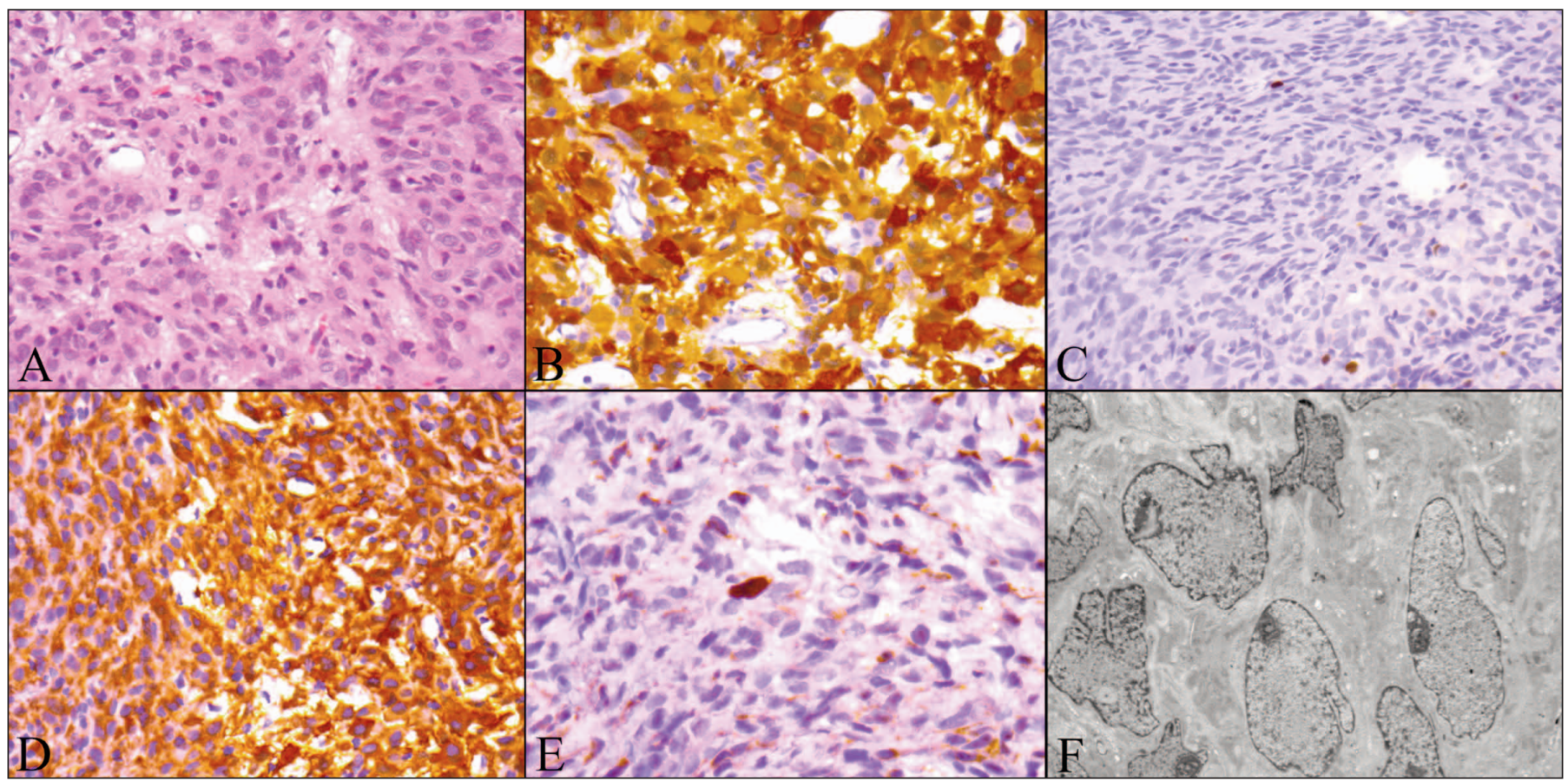

Figure 2: A) H\&E stained section (400x), B) S100 immunostaining (400x) showing diffuse cytoplasmic and nuclear labeling, C) Ki67 immunostaining (400x) labels approximately 1\% of cells, D) VEGF immunostaining (400x) shows diffuse cytoplasmic positivity, E) Topoisomerase II alpha immunostaining (630x) labels rare tumor cell nuclei, F) Electron microscopy (6000x) showing an absence intercellular adhesions.

positive. The tumor cells were negative for neurofilament and synaptophysin. CD34 revealed rich and delicate tumor vasculature. CD45 and CD68 demonstrated admixed reactive inflammatory lymphocytes and macrophages. Immunohistochemistry showed negative results for ACTH, growth hormone, prolactin, TSH, FSH, LH, - subunit, vasopressin, and neurophysin. Ki67 positive cells, labeled with MIB-1 antibody, were estimated to account for $1 \%$ of the tumor cells. Approximately $40 \%$ of tumor cells showed nuclear labeling for p27 with no abnormal cytoplasmic positivity. Topoisomerase II alpha stained approximately $1-5 \%$ of tumor cell nuclei. The findings were diagnostic of pituicytoma.

Part of the tumor was glutaraldehyde-fixed and routinely processed. By electron microscopy this moderately cellular tumor was composed of sheets and groups of small elongated cells with mildly irregular nuclei containing small to moderately developed nucleoli and a small quantity of granular heterochromatin. A thin rim of cytoplasm contained inconspicuous rough endoplasmic reticulum membranes, occasional normal-appearing mitochondria and rare Golgi organelles. The loosely arranged tumor cells have no intercellular adhesions. No secretory granules were identified.

\section{Discussion}

Pituicytomas are rare, generally circumscribed spindle cell neoplasms arising in the neurohypophysis codified as a World Health Organization (WHO) grade I tumor in the 2007 Classification of Tumors of the Central Nervous System ${ }^{1}$. They are radiographically circumscribed, and are $\mathrm{T} 1$ iso-intense with diffuse gadolinium enhancement. Our case showed the classic histologic and immunohistochemical features. It was a spindle cell tumor strongly immnohistochemcially positive for S-100 protein and vimentin with patchy GFAP immunopositivity. Our case also demonstrates the typical ultrastructural features of pituicytoma. The tumor cells had irregular, indented nuclear membranes and showed no evidence of basal lamina, secretory granules, or desmosomes. No fibrous bodies, rarely reported in this tumor, were identified ${ }^{2}$. Pituicytomas are postulated to arise in pituicytes, a specialized glial cell of the posterior pituitary. A recent series studied two cases of pituicytoma with array comparative genomic hybridization and described unique genetic alterations, but interestingly also noted several alterations previously characterized in pituitary adenomas including chromosomal imbalances on $1 \mathrm{p}, 5 \mathrm{p}, 14 \mathrm{q}$, and $22 \mathrm{q}^{3}$. It has also recently been proposed that immunohistochemical staining for EMA may prove useful in distinguishing this tumor from spindle cell oncocytoma of the adenohypophysis, another spindled vascular tumor of the sellar region ${ }^{4}$.

Our case showed strong and diffuse immunohistochemical labeling for VEGF. Vascular endothelial growth factor is a vascular mitogen that increases capillary permeability and plays an important role in glioma neovascularity and peritumoral edema $^{5}$. The VEGF expression has been reported in several tumors of the sella, including pituitary adenomas, and craniopharyngioma. Expression of this marker does not imply aggressive behavior, or increased propensity for invasion. As seen in our case, pituicytomas are highly vascular, and may bleed profusely as a consequence of surgical manipulation. The 
extensive vascularity can hinder the surgeon's ability to achieve a gross total resection and, in the majority of reported cases, only a subtotal resection was possible. This has led some authors to suggest pre-operative embolization in cases where the pathology is known prior to resection. Our results warrant further investigation into VEGF expression in these tumors, and antiVEGF therapy may prove useful in the management of patients with pituicytoma.

Pitucytomas are considered benign, indolent, hormonally inactive neoplasms. They most commonly present with endocrine and visual abnormalities secondary to the mass effect. The slow growth rate of this tumor may account for the lack of diabetes insipidus, despite the typical location in the posterior pituitary. In our case, the tumor was associated with a mild increase in blood prolactin levels as a consequence of "stalkeffect".

In pituicytomas the Ki67 nuclear labeling index is typically low $(<2 \%)$ and mitotic activity is inconspicuous, though rare cases of pituicytoma with increased mitotic activity (5/10 high powered fields) and Ki67 labeling (5\%) have been reported. Immunohistochemical analysis of markers of cellular proliferation in our case are consistent with the low proliferative activity traditionally described in these tumors. Ki67 labeled fewer than $1 \%$ of tumor cell nuclei overall. P27 is a cyclindependant kinase inhibitor. Decreased levels and abnormal nonnuclear localization have been reported to be associated with aggressive behavior. Our case showed no evidence of abnormal cytoplasmic relocalisaiton. Topoisomerase IIalpha (Topo IIalpha) is a marker of cell proliferation. Increased expression of this marker as a percentage of tumor cell nuclei has also been correlated with aggressive behavior (infiltration and metastatic potential) in cases of pituitary adenoma. In our case of pituicytoma no increase in Topo IIalpha nuclear labeling was seen. These additional markers of proliferative activity confirm the currently held view that pituicytomas are slow-growing neoplasms, and that the prognosis is excellent for patients with this rare glial neoplasm of the neurohypophysis.

\section{REFERENCES}

1. Louis DN, Ohgaki H, Wiestler OD, et al. The 2007 WHO classification of tumours of the central nervous system. Acta Neuropathol. 2007 Aug;114(2):97-109.

2. Cenacchi G, Giovenali P, Castrioto C, Giangaspero F. Pituicytoma: ultrastructural evidence of a possible origin from folliculostellate cells of the adenohypophysis. Ultrastruct Pathol. 2001 Jul-Aug;25(4):309-12.

3. Phillips JJ, Misra A, Feuerstein BG, Kunwar S, Tihan T. Pituicytoma: characterization of a unique neoplasm by histology, immunohistochemistry, ultrastructure, and array-based comparative genomic hybridization. Arch Pathol Lab Med. 2010 Jul;134(7):1063-9.

4. Ogiwara H, Dubner S, Shafizadeh S, Raizer J, Chandler JP. Spindle cell oncocytoma of the pituitary and pituicytoma: Two tumors mimicking pituitary adenoma. Surg Neurol Int. 2011;2:116.

5. Berkman RA, Merrill MJ, Reinhold WC, et al. Expression of the vascular permeability factor/vascular endothelial growth factor gene in central nervous system neoplasms. J Clin Invest. 1993 Jan;91(1):153-9. 